\title{
Predicting Plausible Human Purkinje Network Morphology from Simulations
}

\author{
Matthias Lange $^{1}$, Toni Lassila ${ }^{2}$, Alejando F Frangi ${ }^{2}$ \\ ${ }^{1}$ University Of Utah, Salt Lake City, Utah, USA \\ ${ }^{2}$ University of Leeds, Leeds, UK
}

\begin{abstract}
The Purkinje network (PN) gains more clinically importance as it becomes target for pacing in rate control and defibrillation. However, our understanding of the PN morphology arises from animal experiments, which might not transfer to humans. Therefore, we propose an automated computer simulation predicting physiological PN morphologies depending on the heart shape. It starts by generating virtual heart shapes from a statistical shape atlas and generates virtual PNs on the endocardial surface. For the combined virtual models the eikonal equation is solved to estimate the local activation times throughout the myocardium, which then feed forward to an simulation of the 12-lead surface ECG. From the simulated ECG the QRS-complex is compared against a healthy standard QRS-complex, which allows to estimate how physiological a PN morphology is.

In our model, only bundle branch bifurcation points near the base or near the apex result in physiological QRS wave forms. For the right bundle, more physiological QRS waves can be obtained when the branching point is at the apex. Only a minor dependency of the ECG on the heart shape is found. However, a strong correlation between the bundle branch bifurcation points themselves is observed.
\end{abstract}

\section{Introduction}

The Purkinje network (PN) is a fast-conducting network of specialised cardiac cells that transfers the electrical signal arriving at the atrioventricular node to the ventricular muscle. It is well-known that the PN varies between species [1] and in particular from animals to humans. Nevertheless, the majority of morphological and physiological knowledge of the PN arises from animal studies [2] . Studies of human PNs are limited to single subjects [3], and as a result it is unclear to which extent the PN may vary over a large population.

Analysis of the developing heart indicates that the bifurcation point of the bundle branches $(\mathbf{B B})$ and free basal area (Fig. 1) could depend on the heart shape. The shorter the distance a BB runs until it bifurcates the more of the septum is covered by the PN. This influences the first sites of myocardial activation and thereby the synchronicity of the left and right ventricular contraction. The free basal area, detailed below, could additionally influence the total activation time of the heart.

We hypothesise that the PN morphology depends on the heart shape and test this hypothesis with a computational model. We construct an automatic computational pipeline which, based on a parametric model of cardiac shape and PN, predicts whether the electrocardiogram (ECG) shows a single or a double R-spike. The automatic pipline is use to train a surogate model, which allows much faster prediction of physiological PN depending on the heart shape.

\section{Methods}

First, we outline the computational model, and then how to build the surrogate model.

\subsection{The Automatic Pipeline}

The automatic pipeline is formed by the cardiac model and the ECG model. The cardiac model summarizes the models used to estimate the transmembrane potential, where the ECG model calculates the 12 lead ECG from the transmembrane potential.

The cardiac model starts with the creation of a new heart surface mesh based on a statistical shape model (SSM). The SSM is constructed with an algorithm published in [4], from 134 heart surface meshes obtained in the work of [5]. The usage of the SSM allows a parametrisation of the heart shape need to construct the surrogate model. To obtain the final surface mesh the mean mesh is deformed based on the SSM using radial basis functions [6]. On the surface mesh of the heart, a PN is grown using [7] with added control over the coverage of the endocardium by the PN. In detail, there is each one Purkinje parameter for the left and right $\mathrm{BB}$ bifurcation point, and two controlling the free basal area in the left ventricle ( $(\mathbf{V})$ and right ventricle (RV) (Fig 1). From the surface mesh, a volumetric mesh is generated with TetGen ${ }^{1}$ and the myocardial fibre orientation

\footnotetext{
${ }^{1}$ Tetgen:Daunay-based quality tetrahedral meshgenerator,WIAS Berlin
} 


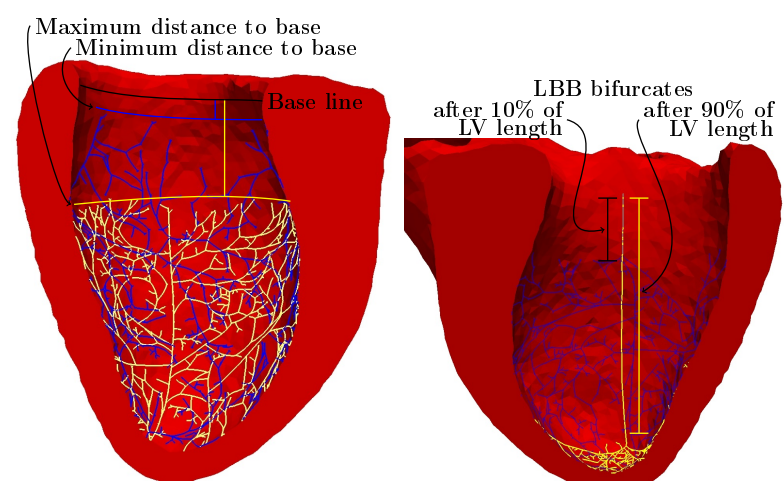

Figure 1. Four parameter controlling the ventricular coverage by the Purkinje network. Left: Free basal area, Right: Bundle branch bifurcation point shown for left ventricle.

is assigned based on a method described in [8]. The geometrical model is used to estimated activation times of the heart with the eikonal model [9], which is solved with the fast marching algorithm [10]. The resulting activation times are converted to time-series of transmembrane potential distributions. For each point in the volume mesh, the activation time is used as the time of upstroke in the action potential. The action potential is sampled from a lookup table of the minimal ionic model [11]. For 150 time-steps, ranging from $0 \mathrm{~ms}$ to $300 \mathrm{~ms}$, the transmembrane potential distribution is exported. The duration of $300 \mathrm{~ms}$ allows all myocytes to complete the depolarization, while the timestep is chosen to capture the upstroke, which takes about $2 \mathrm{~ms}$.

The second part of the automatic pipeline is the ECG model, build from the torso geometry and the forward ECG model. The torso geometry is a fixed mesh consisting of a skin and lung mesh from [12] and the heart surface from the previous model. The geometries are used in the ECG forward solution of

$$
\begin{aligned}
-\nabla \cdot\left(\left(\tilde{\sigma}_{i}+\tilde{\sigma}_{e}\right) \nabla\left(\phi_{T}\right)\right) & =\nabla \cdot\left(\tilde{\sigma}_{i} \nabla(\varphi)\right) & & \forall x \in \Omega_{H} \\
\nabla \cdot\left(\tilde{\sigma}_{T} \nabla\left(\phi_{T}\right)\right) & =0 & & \forall x \in \Omega_{T} \\
\left.\partial_{n} \phi_{T}\right|_{\partial \Omega_{T}} & =0, & &
\end{aligned}
$$

where $\phi_{T}$ is the torso potential, $\varphi$ is the transmembrane potential, and $\tilde{\sigma}_{e}, \tilde{\sigma}_{i}, \tilde{\sigma}_{T}$ are the extracellular, intracellular and torso conductivities. Two domains are distinguished $\Omega=\Omega_{H} \cup \Omega_{T}$ with $\Omega_{H}$ being the domain of the heart and $\Omega_{T}$ the domain of the torso without heart. To solve equation (1) numerically the computer program SCIRun ${ }^{2}$ is used. The solution of the torso potential is obtained for all 150 time-steps of the transmembrane potential exported by the cardiac model. From which the standard 12 lead ECG is derived.

\footnotetext{
${ }^{2}$ SCIRun: A Scientific Computing Problem Solving Environment, Sci-
} entific Computing and Imaging Institute (SCI)

\subsection{The Surrogate Model}

The surrogate model is a function from the 5 dimensional parameter space (4 Purkinje parameters +1 shape parameters) to the cost associated with a double R-spike. To evaluate the presents of a double R-spike, lead II from the ECG is used to fit two Gauss-functions

$$
\begin{aligned}
& g_{s_{1}, \mu_{1}, \sigma_{1}, s_{2}, \mu_{2}, \sigma_{2}}(t)= \\
& \underbrace{\frac{s_{1}}{\sqrt{2 \sigma_{1}^{2} \pi}} \exp \left(\frac{\left(x-\mu_{1}\right)^{2}}{-2 \sigma_{1}^{2}}\right)}_{N_{1}}+\underbrace{\frac{s_{2}}{\sqrt{2 \sigma_{2}^{2} \pi}} \exp \left(\frac{\left(x-\mu_{2}\right)^{2}}{-2 \sigma_{2}^{2}}\right)}_{N_{2}} .
\end{aligned}
$$

To fit $g(t)$, the R-spike is centred at the origin $E C G(0)=$ $\max (E C G)$, then the $L_{2}$ error between the signal and $g(t)$ is minimised with a constrained minimisation. A penalty term $r_{1}$ is used to prevent both Gauss-function at the origin

$$
\begin{array}{cl}
E\left(s_{1}, \mu_{1}, \sigma_{1}, s_{2}, \mu_{2}, \sigma_{2}\right)= & \\
& \int(E C G(t)-g(t))^{2} d t+\underbrace{\left|\mu_{1}-\mu_{2}\right|^{-1}}_{r_{1}}, \\
& 0 \geq \frac{s_{2}}{\sigma_{2}}-\frac{s_{1}}{\sigma_{1}} \\
\text { s.t. } & \mu_{1} \in[-3,3], \sigma_{1} \in[10 ., 40], s_{1} \in[-20,200] \\
& \mu_{2} \in[-50,50], \sigma_{2} \in[0.1,50], s_{2} \in[-0.1,30] .
\end{array}
$$

The constrains ensures that $N_{1}$ is the largest spike. The domain of the function $E$ allows a negative amplitude, because the R-spike can become negative for some Purkinje parameter values.

The cost function is generated from the six features $\left(s_{1}, \sigma_{1}, \mu_{1}, s_{2}, \sigma_{2}, \mu_{2}\right)$ obtained during fitting

$$
\begin{aligned}
& f_{1}\left(s_{1}, s_{2}, \sigma_{1}, \sigma_{2}\right):=\operatorname{sig}\left(100 \frac{s_{2} \sigma_{1}}{s_{1} \sigma_{2}}, 20\right) \\
& f_{2}\left(\mu_{1}, \mu_{2}\right):=\operatorname{sig}\left(\left(\left(\mu_{1}-\mu_{2}\right) / \mu_{2}\right)^{2}, 2\right) \\
& f_{3}\left(\sigma_{1}\right):=\operatorname{sig}\left(\sigma_{1}, 20\right) \\
& \operatorname{Cost}\left(s_{1}, \mu_{1}, \sigma_{1}, s_{2}, \mu_{2}, \sigma_{2}\right):=f_{1} \cdot f_{2}+0.5 f_{3}
\end{aligned}
$$

with the Sigmoid function

$$
\operatorname{sig}\left(x, x_{0}\right):=1 / 1+\exp \left(x_{0}-x\right) .
$$

The cost function compares the ratio of the amplitude of a possible double spike $\left(f_{1}\right)$, measures the spike separation $\left(f_{2}\right)$ and the absolute amplitude of the main spike $\left(f_{3}\right)$. The cost becomes smaller if the double peaks get closer to each other and has a minimum if only one peak is detected.

The cost is used in the sparse grid algorithm by A. Klimke [13], to train a piecewise linear sparse grid interpolant from the 5 model paramets to the cost

$$
S \text { (4 Purkinje Parameter, } 1 \text { Shape Parameter }) \mapsto \text { Cost. }
$$




\section{Results}

In the following, we plot the energy function while varying two parameters of the automatic pipeline. All other parameters are keep fixed at mid range. For the heart shape the range is two standard deviation $(\sqrt{\sigma})$ from the mean shape, while the bifurcation point is at $10 \%$ to $90 \%$ of the left or right ventricular length. In the resulting plot, the smaller the energy is, the more physiological is the simulated QRS.

For the left BB a bifurcation point (Fig. 2) near the base or near the apex would be favoured by the computational model. The experiments with shape deformation in the negative direction of mode one yield an ECG with a double $\mathrm{R}$-spike, for all tested bifurcation points of the left BB.

In case of the right $\mathrm{BB}$ bifurcation point, the results are more conclusive and strongly favour a bifurcation point close to the apex. As for the left BB bifurcation a slight dependency on the heart shape is observed. However, for all shapes the same bifurcation point is favoured.

A strong correlation between the left and right $\mathrm{BB}$ bifurcation point exists (Fig. 3). As already seen in Fig. 2, for both BBs exists parameter values for which the cost becomes small. However, only if both bifurcation parameters are chosen optimally an ECG with a single R-spike is produced.

The experiment in variation of the free basal area in LV and RV has shown a smaller influence on the ECG (Fig. 4). Further, there is no dependency on the shape to be noted.

\section{Discussion}

With the help of computational simulations, we observed strong dependencies between the BB bifurcation points and the resulting ECGs. A particular configuration of the right BB bifurcation point was observed over a range of cardiac shapes and is in agreement with literature [2]. The strong correlation be between left and right BB bifurcation point was also expected, because bifurcation points determinate the synchronous activation of LV and RV. Our model confirmed this intuitive observation.

Our model showed little dependency of the cost function on the heart shape. This could be because the resulting ECG does not change, but also because our cost function is not sensitive to the shape change. A further reason could be that left and right BB bifurcation points have greater influence on the ECG then the heart shape and need to optimised before seeing a dependency of PN morphology on the heart shape.

Similar observations can be made for the free basal area, which has nearly no influence on the resulting ECG. Varying the free basal area in the RV can reduce the separation of the double spike. This could be related to the longer activation time in the RV if the free basal area increases.

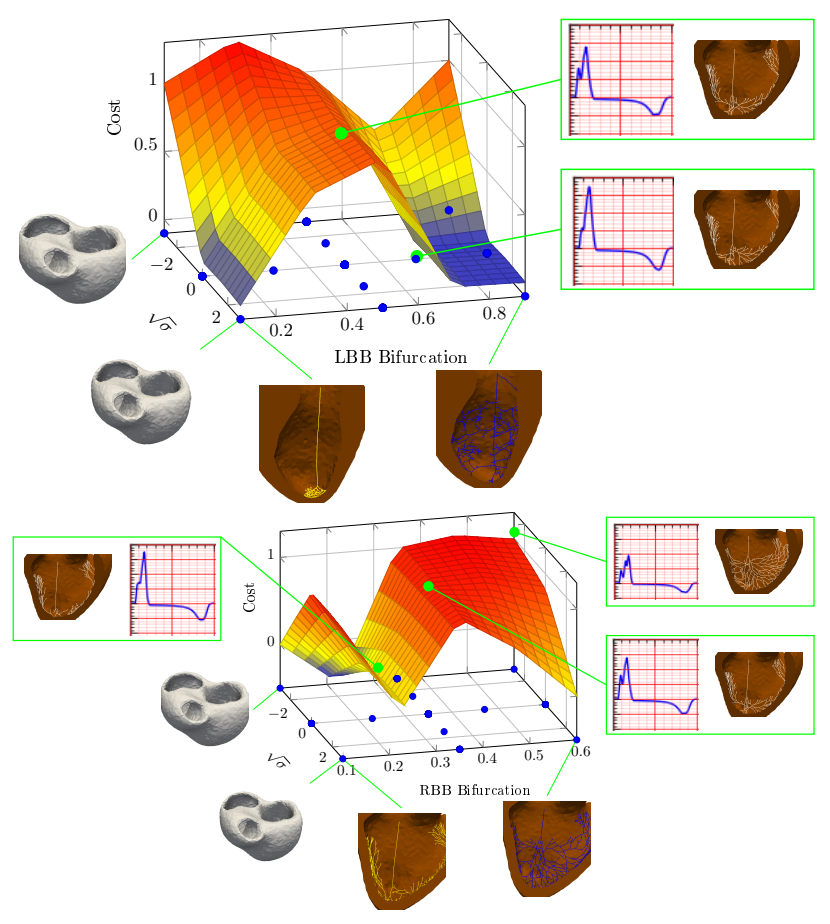

Figure 2. Cost function in relation to bundle branch bifurcation point and shape.

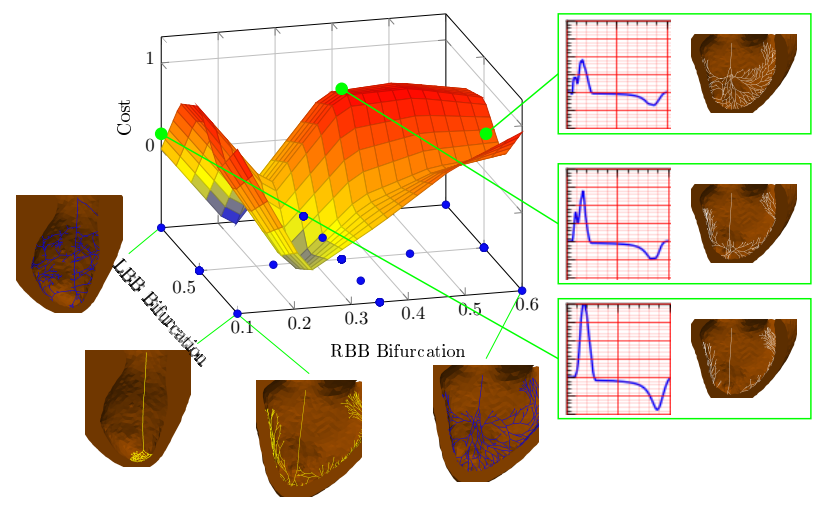

Figure 3. Cost function with respect to bundle branch bifurcation points. Exhibiting the most sensitivity compared to the other experiments.

However, for all experiments the separation persisted.

As do all studies, this study has limitations. One is the current sparse grid, which chose the middle of the parameter range as a reference point. This means, that for many simulations non optimal bifurcation parameters are used. Taken the results for this study into account, it would be better if the sparse grid algorithm first searched for the correct left and right $\mathrm{BB}$ bifurcation point and then explored other parameter dependencies. Otherwise, a bias towards the chosen bifurcation points exist. The second limitation is the restriction to the first mode of shape variation in this 


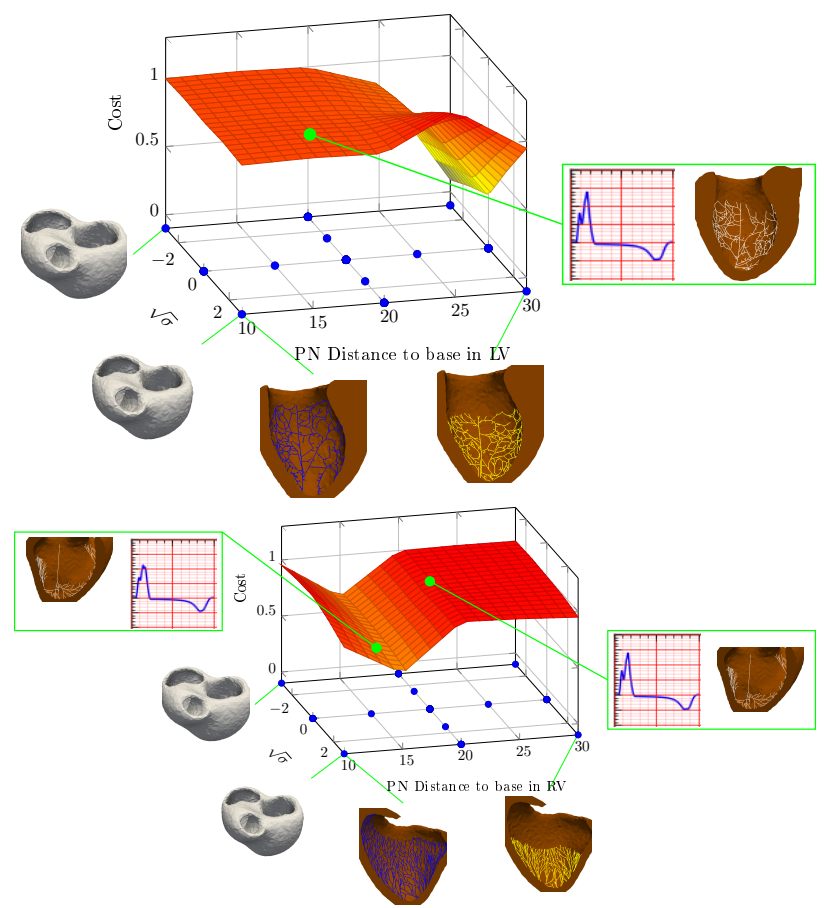

Figure 4. Cost function in relation to free basal area and shape.

study. Using more shape variation modes could provide more insight in the shape dependency of the PN. Equally, the PN parameter could include the density of PurkinjeMyocardial-Junctions. Furthermore, this study uses only the lead II of the ECG. These means the PN optimisation process evaluates only one of three spatial axes.

\section{Conclusions}

In conclusion, our simulation-based study showed that the bifurcation points of the left and right BB have a major impact on the QRS complex. Both parameters need to be tuned simultaneously to obtain a single-peak QRS. On the other hand, the simulation indicated minor importance of the free basal area for the correct formation of the ECG. Overall, the experiments do not confirm the hypothesises that the PN morphology depends on the heart shape, for the first mode of variation in the SSM.

Our systematic exploration of the ECG as a function of cardiac structure was possible because the heart shape was parametrised through a SSM and the PNs could algorithmically-generated. This allowed to simulate specific virtual hearts and rule out certain configurations of the BB bifurcation points as unphysiological. This idea should be further developed to consider ways of identifying the PN from ECG measurements in an in-vivo setting.

\section{References}

[1] Pennisi DJ, Rentschler S, Gourdie RG, Fishman GI, Mikawa T. Induction and patterning of the cardiac conduction system. Int J Dev Biol 2002;46(6):765-75.

[2] Atkinson A, Inada S, Li J and Tellez JO, Yanni J, Sleiman R, Allah EA, Anderson RH, Zhang H, Boyett MR, Dobrzynski $\mathrm{H}$. Anatomical and molecular mapping of the left and right ventricular His-Purkinje conduction networks. J Mol Cell Cardiol 2011;51(5):689-701.

[3] Tawara S. Das Reizleitungssystem des Saeugetierherzens. Jena: von Gustav Fischer, 1906.

[4] Ravikumar N, Gooya A, Cimen S, Frangi AF, Taylo ZA. A multi-resolution T-mixture model approach to robust groupwise alignment of shapes. Springer International Publishing. ISBN 978-3-319-46726-9, 2016; 142-149.

[5] Hoogendoorn C, Duchateau N, Sánchez-Quintana D, Whitmarsh T, Sukno FM, De Craene M, Lekadir K, Frangi AF. A high-resolution atlas and statistical model of the human heart from multislice CT. IEEE Trans Med Imaging 2013; 32(1):28-44.

[6] Gooya A, Davatzikos C, Frangi AF. A bayesian approach to sparse model selection in statistical shape models. SIAM J Imaging Sci 2015;8(2):858-887.

[7] Lange M, Di Marco LY, Lekadir K, Lassila T, Frangi AF. Protective role of false tendon in subjects with left bundle branch block: A virtual population study. PLOS ONE 2016; 11(1):1-23.

[8] Rossi S, Lassila T, Ruiz-Baier R, Sequeira A, Quarteroni A. Thermodynamically consistent orthotropic activation model capturing ventricular systolic wall thickening in cardiac electromechanics. Eur J Mech ASolids 2013;48:129_ 142.

[9] Keener JP, Sneyd J. Mathematical physiology. New York: Springer, 1998.

[10] Pashaei A, Romero D, Sebastian R, Camara O, Frangi AF. Fast multiscale modeling of cardiac electrophysiology including Purkinje system. IEEE Trans Biomed Eng 2011; 58(10):2956-60.

[11] Bueno-Orovio A, Cherry EM, Fenton FH. Minimal model for human ventricular action potentials in tissue. J Theor Biol 2008;253(3):544-60.

[12] MacLeod RS, Johnson CR, Ershler PR. Construction of an inhomogeneous model of the human torso for use in computational electrocardiography, volume 13. pt 2 edition. Publ by IEEE, 1991; 688-689.

[13] Klimke A. Sparse grid interpolation toolbox - user's guide. Technical Report IANS report 2007/017, University of Stuttgart, 2007.

Address for correspondence:

Matthias Lange

Cardiovascular Research and Training Institute

95 South 2000 East,

Salt Lake City, Utah 84112

Matthias.lange@Utah.edu 